\title{
Synthesis and Anticonvulsant Activity of Various Mannich and Schiff Bases of 1,5-Benzodiazepines
}

\author{
Surendra N. Pandeya and Neha Rajput \\ Department of Pharmaceutical Sciences, Saroj Institute of Technology and Management, Sultanpur Road, \\ Lucknow 226002, India \\ Correspondence should be addressed to Neha Rajput,nr.17neha@gmail.com
}

Received 6 May 2012; Revised 6 September 2012; Accepted 28 September 2012

Academic Editor: Jochen Lehmann

Copyright (๑) 2012 S. N. Pandeya and N. Rajput. This is an open access article distributed under the Creative Commons Attribution License, which permits unrestricted use, distribution, and reproduction in any medium, provided the original work is properly cited.

\begin{abstract}
Benzodiazepines have a various behavioral effects in addition to their anxiolytic action. There is every reason to believe that the $\mathrm{BZ} / \mathrm{GABA}$ receptor complex is involved in these effects, since GABAmimetic manipulations modify the effect of BZ in tests of convulsive activity, motor function, and appetitive behavior. 1,5-Benzodiazepines are biologically important molecules and are extensively used clinically as analgesic, hypnotic, sedative, and antidepressive agents. Hence, 1,5-Benzodiazepines were synthesized by condensation of o-phenylenediamine and ketones, for example, cyclohexanone and acetone in presence of sulfated zirconia (catalyst). Mannich bases were synthesized with acetophenone, p-nitroacetophenone, p-chloroacetophenone, and formaldehyde. Schiff bases were synthesized using Mannich base of 1,5-benzodiazepines with p-chloroaniline and p-chlorophenylsemicarbazide in the presence of glacial acetic acid. All the synthesized compounds were characterized by ${ }^{1} \mathrm{H}$ NMR and IR spectral analyses. All the synthesized derivatives were evaluated at the dose of $30 \mathrm{mg} / \mathrm{kg}$ b.w for anticonvulsant activity by isoniazid induced convulsion model, and the compounds NBZD-3 and NBZD-8 were found to be the most active among all compounds. Among all the synthesized derivatives, compounds NBZD-13 and NBZD-17 were found to be the most active among all compounds using thiosemicarbazide induced model. Although NBZD-8, NBZD-10, and NBZD-18 are the compounds which had shown good anticonvulsant activity and have an advantage over that, they were not sedative.
\end{abstract}

\section{Introduction}

A benzodiazepine is a psychoactive drug whose core chemical structure is the fusion of a benzene ring and a diazepine ring. The first benzodiazepine, chlordiazepoxide (Librium), discovered accidentally by Leo Sternbach in 1955 and made available in 1960 by Hoffmann La Roche, which has also marketed diazepam (Valium) since 1963 [1]. 1,5-Benzodiazepines constitute an important class of psychopharmaca [2], in particular as tranquilizers and also as potent Virucides and nonnucleoside inhibitors of HIV-1 reverse transcriptase [3].

Benzodiazepine has a traditional place in antiepileptic therapy. The clinical use of BZDs can be divided into two categories. First, in the acute treatment of seizures as drugs of choice in status epilepticus and also in some cases of febrile seizures. Second, the BZDs are utilized in long-term therapy of certain seizures types primarily in the pediatrics' population [4].

There are some differences between the effects of 1,5and 1,4-benzodiazepines. A greater therapeutic potential and lower incidence of side effects were described for 1,5BZDs when compared to 1,4 -BZDs. $1,5-\mathrm{BZD}$ is used as adjuvant therapy in resistant cases of epilepsies [5]. BZDs exhibit potent anticonvulsant actions in a wide variety of animal seizures models. They are particularly effective against seizures induced by electroshock [6], and various chemoconvulsants, in kindled seizures and in absence seizures [7].

Beside this, 1,5-benzodiazepines show antifungal, antibacterial [8], antifeedant [9], anti-inflammatory analgesic [10], and anticonvulsant activities [11]. The benzodiazepines nucleus is a well-studied traditional pharmacophoric scaffold 
that has emerged as a core structure unit of various biological activities [12].

Although, the first benzodiazepine was introduced as a drug nearly 35 years ago, the research in this area is still very active and is directed towards the synthesis of compounds with enhanced pharmacological activity [13]. The chemical structure of the benzodiazepines seems at first sight to be unique among the various types of central depressant drugs [14]. 1,5-Benzodiazepines derivatives show a large number of pharmacological properties such as they acted as sedatives [15], Cerebrovasodialators [16], neuroleptics [17], antispasmodic [18], anticonvulsant [19], tranquilizing agents [20], antibacterial [21], and psoriasis [22] and used for the treatment of small pox [23].

\section{Experimental Procedure}

Starting material and reagents were procured from commercial chemical suppliers. All the chemicals and solvents used were of laboratory grade. Melting points were determined in open capillary tubes and are uncorrected. IR spectra $(\mathrm{KBr}$, $\mathrm{cm}^{-1}$ ) were recorded on Perkin Elmer Spectrometer, $1 \mathrm{H}$ NMR- $(\delta$, ppm) spectra were recorded on a Brucker $300 \mathrm{MHz}$ NMR spectrometer using TMS as an internal standard. The purity of compounds and progress of the reaction were checked by TLC using silica gel-G as adsorbent.

\subsection{Procedure for Preparation of Fused Ring Benzodiazepine Nucleus}

2.1.1. Synthesis of Fused Ring Benzodiazepine Nucleus. Synthesis of fused ring benzodiazepines in presence of sulfated zirconia involves 2 steps which are as follows [9].

Preparation of Catalyst. $25 \mathrm{gm}$ of zirconium oxychloride was dissolved in doubly distilled water ( $\mathrm{pH}-2)$. Dilute aq. ammonia was then added dropwise from a burette with vigorous $(\mathrm{pH}=8)$. Precipitate was washed with distilled water several times and dried for $24 \mathrm{~h}$. Sample was ground to fine powder and immersed in an $0.5 \mathrm{M} \mathrm{H} 2 \mathrm{SO} 4$ solution $(30 \mathrm{~mL})$ for $30 \mathrm{~min}$. Excess water was evaporated on water bath, and the resulting sample was oven dried.

Synthesis of Benzodiazepines. $1: 2.5$ mole ratio mixture of ophenylenediamine and ketone (cyclohexanone (Scheme 1)) with catalytic amount of sulfated zirconia was taken in round bottom flask (RBF) with stirring at ambient condition for 2$3 \mathrm{~h} .10 \mathrm{~mL}$ of $\mathrm{CH} 2 \mathrm{Cl} 2$ was added to reaction mixture, and catalyst was recovered by filtration.

\subsection{Procedure for Preparation of Mannich Base Derivatives}

2.2.1. Synthesis of Various Mannich Base Derivatives of Fused Ring Benzodiazepine (Scheme 2). Equimolar quantity of fused ring benzodiazepine (NBZD-1, 0.01 M), formaldehyde, and various acetophenones (i.e., acetophenone, pnitroacetophenone, and p-chloroacetophenone) were taken in RBF, and mixture was refluxed for $2.30 \mathrm{~h}$. Completion of reaction was monitored by TLC analysis for several times. Then, reaction mixture was evaporated on water bath and dried. Melting point, Rf value, and \% yield were noted. Various Mannich base derivatives are shown in Scheme 2.

\subsection{Procedure for Preparation of Schiff Base Derivatives}

2.3.1. Synthesis of Various Schiff Base Derivatives of Fused Ring Benzodiazepines. Equimolar quantities of Mannich base derivatives (0.01 M, NBZD-3, NBZD-4, and NBZD5), in individual reactions, were dissolved in glacial acetic acid and added with p-chloroaniline (Scheme 3) or pchlorophenylsemicarbazide (Scheme 4) and taken in RBF and mixture was refluxed for $3 \mathrm{~h}$, respectively. Completion of reaction was monitored by TLC analysis for several times in chloroform: ethanol $(1: 1)$. Then, reaction mixture was evaporated on water bath and dried. Melting point, $\mathrm{Rf}$ value, and $\%$ yield were noted.

\subsection{Procedure for Preparation of 1,5-Benzodiazepine Nucleus}

2.4.1. Synthesis of 1,5-Benzodiazepine Nucleus. Synthesis of 1,5-benzodiazepines in presence of sulphated zirconia involves 2 steps which are as follows [9].

Synthesis of Benzodiazepines. $1: 2.5$ mole ratio mixture of o-phenylenediamine and acetone (Scheme 5) with catalytic amount of sulfated zirconia was taken in round bottom flask (RBF) with stirring at ambient condition for $2-3 \mathrm{~h} .10 \mathrm{~mL}$ of $\mathrm{CH} 2 \mathrm{Cl} 2$ was added to reaction mixture, and catalyst was recovered by filtration.

\subsection{Procedure for Preparation of Mannich Base Derivatives}

2.5.1. Synthesis of Various Mannich Base Derivatives of 1,5-Benzodiazepine (Scheme 6). Equimolar quantities of fused ring benzodiazepine (NBZD-12, $0.01 \mathrm{M}$ ), formaldehyde, and various acetophenones (i.e., acetophenone, pnitroacetophenone, and $\mathrm{p}$-chloroacetophenone) were taken in RBF, and mixture was refluxed for $2.30 \mathrm{~h}$. Completion of reaction was monitored by TLC analysis for several times. Then, reaction mixture was evaporated on water bath and dried. Melting point, Rf value, and \% yield were noted. Various Mannich base derivatives are shown in Scheme 6.

\subsection{Procedure for Preparation of Schiff Base Derivatives}

2.6.1. Synthesis of Various Schiff Base Derivatives of 1,5Benzodiazepines. Equimolar quantities of Mannich base derivatives (0.01 M, NBZD-13, NBZD-14, and NBZD-15), in individual reactions, were dissolved in glacial acetic acid and added with p-chloroaniline (Scheme 7) or pchlorophenylsemicarbazide (Scheme 8), and were taken in $\mathrm{RBF}$, and mixture was refluxed for $3 \mathrm{~h}$, respectively. Completion of reaction was monitored by TLC analysis for several times in chloroform: ethanol $(1: 1)$. Then, reaction mixture was evaporated on water bath and dried. Melting point, Rf value, and $\%$ yield were noted. 
<smiles>Nc1ccccc1N</smiles>

O-phenylenediamine<smiles>O=C1CCCCC1</smiles>

Cyclohexanone<smiles>c1ccc2c(c1)N=C1CCCCC1C1(CCCCC1)N2</smiles>

NBZD-1

Scheme 1: Synthesis of fused ring benzodiazepine.

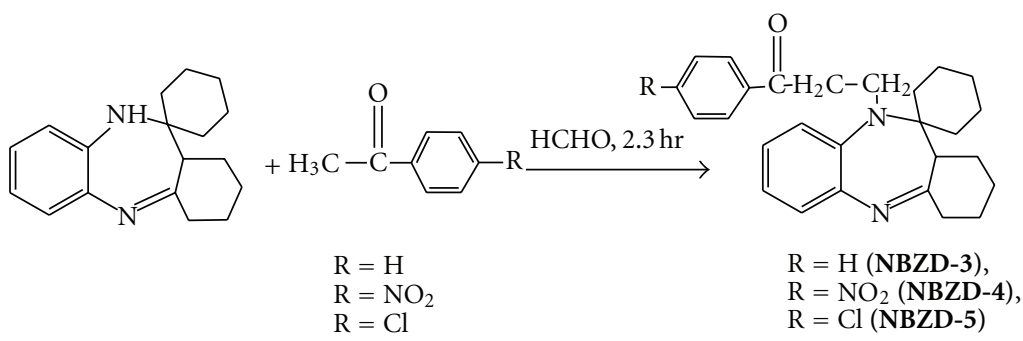

NBZD-1

Ketone

Mannich bases

Scheme 2: Synthesis of various Mannich base derivatives of fused ring benzodiazepines.

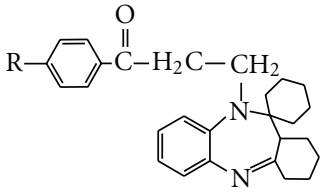

$\mathrm{R}=\mathrm{H}($ NBZD-3),

$\mathrm{R}=\mathrm{NO}_{2}($ NBZD-4)

$\mathrm{R}=\mathrm{Cl}$ (NBZD-5)

Mannich base of 1,5-BZD
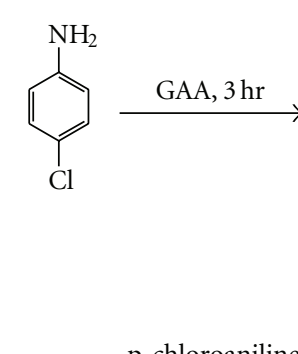

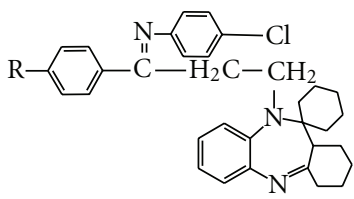

$\mathrm{R}=\mathrm{H}(\mathrm{NBZD}-6)$,

$\mathrm{R}=\mathrm{NO}_{2}$ (NBZD-7)

$\mathrm{R}=\mathrm{Cl}(\mathbf{N B Z D}-\mathbf{8})$

Scheme 3: Synthesis of Schiff base derivative of fused ring benzodiazepine from p-chloroaniline.<smiles>[Y]c1ccc(C(=O)CC)cc1</smiles>

$\mathrm{R}=\mathrm{H}(\mathrm{NBZD}-3)$,

$\mathrm{R}=\mathrm{NO}_{2}(\mathrm{NBZD}-4)$

$\mathrm{R}=\mathrm{Cl}(\mathrm{NBZD}-5)$

Mannich base of fused ring BZD p-chlorophenylsemicarbazide Schiff base of fused ring BZD<smiles>CC(C)CC(=O)Nc1ccccc1</smiles>

$\mathrm{Cl}$<smiles>[R]c1ccc(/C(C)=N/NC(=O)Nc2ccc(Cl)cc2)cc1</smiles>

$\mathrm{R}=\mathrm{H}($ NBZD-9),

$\mathrm{R}=\mathrm{NO}_{2}$ (NBZD-10),

$\mathrm{R}=\mathrm{Cl}$ (NBZD-11)

Scheme 4: Synthesis of Schiff base derivative of fused ring benzodiazepine from p-chlorophenylsemicarbazide.<smiles>Nc1ccccc1N</smiles>

O-phenylenediamine

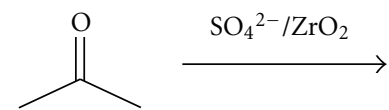

Acetone<smiles>CC1=Nc2ccccc2NC(C)(C)C1</smiles>

NBZD-12

Scheme 5: Synthesis of 1,5-benzodiazepine (NBZD-12). 


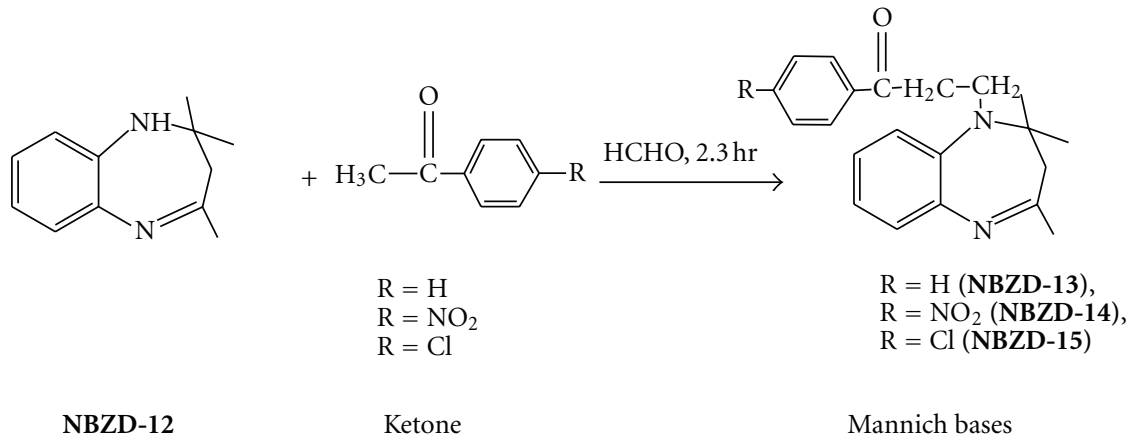

Scheme 6: Synthesis of various Mannich base derivatives of 1,5-benzodiazepines.

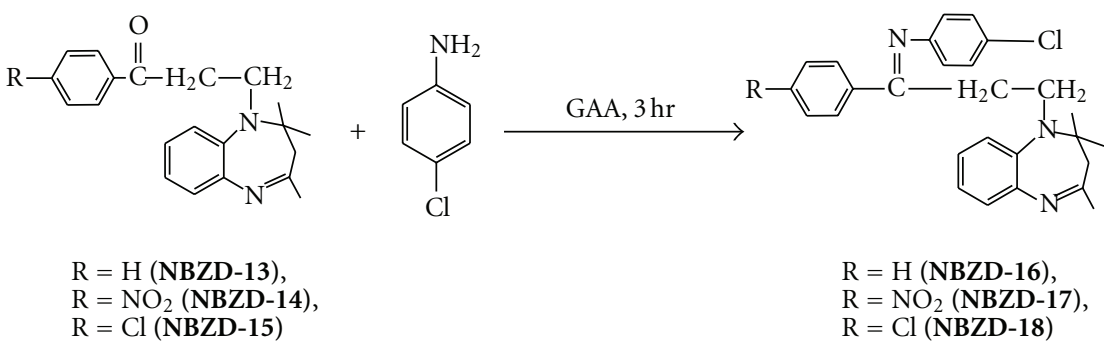

Mannich base of 1,5-BZD p-chloroaniline Schiff base of 1,5-BZD

Scheme 7: Synthesis of Schiff base derivative of 1,5-benzodiazepine from p-chloroaniline.

\section{Anticonvulsant Activity}

3.1. Chemical Induced Model. Ten mice of either sex with a weight of $22-25 \mathrm{~g}$ were treated with the test compounds (30 mg/kg b.w) or the standard (e.g., diazepam $10 \mathrm{mg} / \mathrm{kg}$ b.w) by i.p. administration. Controls received the vehicle only. $30 \mathrm{~min}$ after i.p. treatment, the animals were injected with a subcutaneous dose of $(300 \mathrm{mg} / \mathrm{kg}$, s.c) isoniazid, thiosemicarbazide $(20 \mathrm{mg} / \mathrm{kg}$, s.c). The occurrence of clonic seizures, tonic seizures, and death or recovery were recorded after $0.5 \mathrm{hr}, 1 \mathrm{hr}, 2 \mathrm{hr}$, and $4 \mathrm{hr}$, respectively, for isoniazid induced convulsion (Table 3 ) and also Thiosemicarbazide induced convulsion (Table 4).

3.2. Neurotoxicity Screen. Minimal motor impairment was measured in mice by the rotarod test. The mice were trained to stay on an accelerating rotarod that rotates at 20 revolutions per minute. The rod diameter was $3.2 \mathrm{~cm}$. Neurotoxicity was indicated by the inability of the animal to maintain equilibrium on the rod for at least $1 \mathrm{~min}$ in each of the three trials. The dose at which the animals were unable to grasp the rotarod was determined (Tables 3 and 4).

DOSE: Test drug: $30 \mathrm{mg} / \mathrm{Kg}$ b.w i.p.

3.3. Sedative-Hypnotic Activity. This test was performed with the test substances in a dose of $30 \mathrm{mg} / \mathrm{kg}$ by phenobarbitone induced narcosis in rats. The compounds in PEG (polyethylene glycol) were administered i.p to a group of six rats. After $30 \mathrm{~min}$, rats were then placed on their back and loss of righting reflex was taken as onset of sleep. The time taken by the rats to awake was noted. A control was also performed after pretreatment with test substances vehicle (PEG) and injected phenobarbitone (Table 5).

\section{Results}

4.1. Physicochemical Characterization. See Table 1.

\subsection{Elemental Analysis. See Table 2.}

\subsection{Representative Spectral Analysis}

(1) 10-Spirocyclohexane-1,2,3,9,10,10a-hexahydrobenzo[b]cyclohexane $[\mathrm{e}][1,4]$ diazepine (NBZD-1):

${ }^{1} \mathrm{H}$ NMR (300 MHz, $\left.\delta\right): \mathrm{CH}(\mathrm{m}, 6.4-7.0,4 \mathrm{H}$, phenyl), $\mathrm{NH}(\mathrm{s}, 4.1,1 \mathrm{H}), \mathrm{CH}_{2}$ (m, 1.2-1.6, 18H, cyclohexane), $\mathrm{CH}(\mathrm{s}, 2.7,1 \mathrm{H}$, diazepine ring).

IR (KBr): $\mathrm{NH}\left(\mathrm{Ar}, \quad 3030 \mathrm{~cm}^{-1}, \mathrm{str}\right), \mathrm{CH}(\mathrm{Ar}$, $3180 \mathrm{~cm}^{-1}$, str), $\mathrm{CH}\left(\mathrm{Ar}, 800 \mathrm{~cm}^{-1}\right.$, bend $), \mathrm{C}=\mathrm{N}$ $\left(1618 \mathrm{~cm}^{-1}, \mathrm{Str}\right) \mathrm{CH}_{2}\left(1490 \mathrm{~cm}^{-1}, \mathrm{str}\right), \mathrm{C}-\mathrm{C}(\mathrm{Ar}$, $\left.1600 \mathrm{~cm}^{-1}\right), \mathrm{C}=\mathrm{C}\left(\mathrm{Ar}, 1410,1500,1580 \mathrm{~cm}^{-1}\right)$.

(2) 1-Phenyl-3-(10-spirocyclohexane-1,2,3,9,10,10a-hexahydrobenzo[b]cyclohexane $[\mathrm{e}][1,4]$ diazepine-1-yl) propan-1-one (NBZD-3):

${ }^{1} \mathrm{H}$ NMR $(300 \mathrm{MHz}, \delta): \mathrm{CH}(\mathrm{m}, 7.3-7.9,5 \mathrm{H}$, acetophenone), $\mathrm{CH}_{2}\left(\mathrm{~s}, 2.8,2 \mathrm{H},-\mathrm{COCH}_{2}\right), \mathrm{CH}_{2}$ (s, 3.5, 


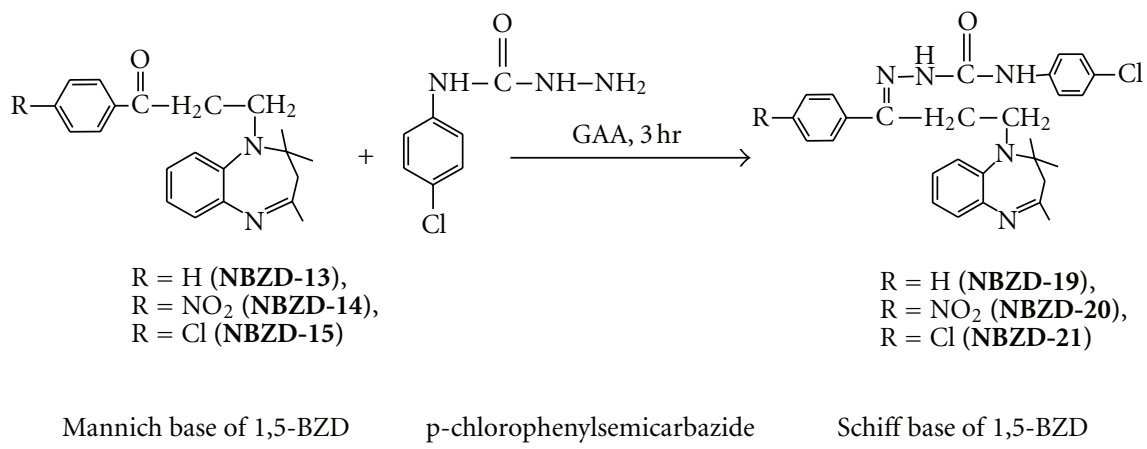

SCHEME 8: Synthesis of Schiff base derivative of 1,5-benzodiazepine from p-chlorophenylsemicarbazide.

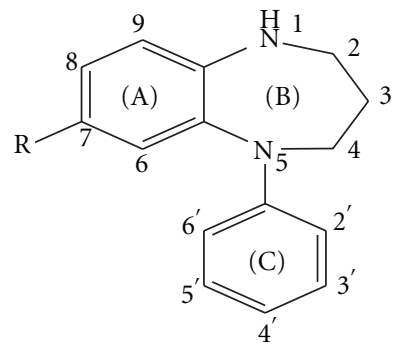

General structure of 1,5-benzodiazepine

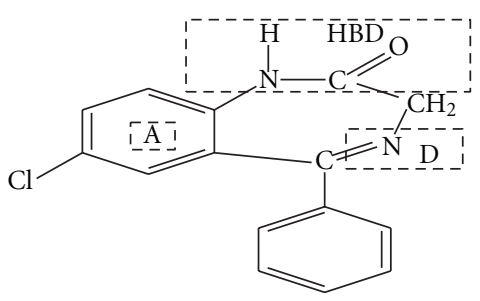

$\mathrm{N}$-desmethyl diazepam

$\mathrm{A}=$ hydrophobic unit, $\mathrm{HBD}=$ hydrogen

bonding domain, $\mathrm{D}$ = electron donor

SCHEME 9

TABLE 1: Physicochemical data of synthesized compounds.

\begin{tabular}{|c|c|c|c|c|c|c|}
\hline Compound code & Molecular formula & Molecular weight & Melting point $\left({ }^{\circ} \mathrm{C}\right)$ & $\%$ yield & Rf value & $\log P$ value \\
\hline NBZD-1 & $\mathrm{C}_{18} \mathrm{H}_{24} \mathrm{~N}$ & 268.19 & 110 & 76.48 & 0.689 & 4.25 \\
\hline NBZD-3 & $\mathrm{C}_{27} \mathrm{H}_{32} \mathrm{~N}_{2} \mathrm{O}$ & 400.25 & 92 & 85.7 & 0.78 & 6.25 \\
\hline NBZD-4 & $\mathrm{C}_{33} \mathrm{H}_{36} \mathrm{~N}_{3} \mathrm{Cl}$ & 510.12 & 132 & 83.3 & 0.73 & 6.15 \\
\hline NBZD-5 & $\mathrm{C}_{34} \mathrm{H}_{28} \mathrm{ClN}_{5} \mathrm{O}$ & 568.15 & 140 & 72 & 0.83 & 6.81 \\
\hline NBZD-6 & $\mathrm{C}_{27} \mathrm{H}_{31} \mathrm{~N}_{3} \mathrm{O}_{3}$ & 445.55 & 98 & 84.3 & 0.63 & 8.93 \\
\hline NBZD-7 & $\mathrm{C}_{27} \mathrm{H}_{31} \mathrm{ClN}_{2} \mathrm{O}$ & 435.00 & 98 & 79.2 & 0.82 & 6.70 \\
\hline NBZD-8 & $\mathrm{C}_{33} \mathrm{H}_{35} \mathrm{ClN}_{4} \mathrm{O}_{2}$ & 554.24 & 130 & 67.2 & 0.57 & 9.49 \\
\hline NBZD-9 & $\mathrm{C}_{34} \mathrm{H}_{37} \mathrm{ClN}_{6} \mathrm{O}_{3}$ & 612.26 & 126 & 56.98 & 0.52 & 8.07 \\
\hline NBZD-10 & $\mathrm{C}_{33} \mathrm{H}_{35} \mathrm{Cl}_{2} \mathrm{~N}_{3}$ & 543.22 & 136 & 65.8 & 0.54 & 9.56 \\
\hline NBZD-11 & $\mathrm{C}_{34} \mathrm{H}_{37} \mathrm{Cl}_{2} \mathrm{~N}_{5} \mathrm{O}$ & 601.24 & 130 & 66 & 0.66 & 8.62 \\
\hline NBZD-12 & $\mathrm{C}_{12} \mathrm{H}_{16} \mathrm{~N}_{2}$ & 188.60 & 96 & 72 & 0.68 & 2.22 \\
\hline NBZD-13 & $\mathrm{C}_{21} \mathrm{H}_{24} \mathrm{~N}_{2} \mathrm{O}$ & 320.3 & 98 & 72.32 & 0.72 & 4.22 \\
\hline NBZD-14 & $\mathrm{C}_{21} \mathrm{H}_{23} \mathrm{~N}_{3} \mathrm{O}_{3}$ & 365.43 & 102 & 92.1 & 0.72 & 4.83 \\
\hline NBZD-15 & $\mathrm{C}_{21} \mathrm{H}_{24} \mathrm{~N}_{2} \mathrm{O}$ & 320.43 & 110 & 57.6 & 0.75 & 4.78 \\
\hline NBZD-16 & $\mathrm{C}_{21} \mathrm{H}_{23} \mathrm{ClN}_{2} \mathrm{O}$ & 354.87 & 108 & 82.9 & 0.73 & 6.90 \\
\hline NBZD-17 & $\mathrm{C}_{27} \mathrm{H}_{28} \mathrm{ClN}_{3}$ & 429.98 & 140 & 90.4 & 0.78 & 7.20 \\
\hline NBZD-18 & $\mathrm{C}_{27} \mathrm{H}_{27} \mathrm{ClN}_{4} \mathrm{O}_{2}$ & 474.98 & 136 & 64.06 & 0.75 & 7.46 \\
\hline NBZD-19 & $\mathrm{C}_{27} \mathrm{H}_{27} \mathrm{Cl}_{2} \mathrm{~N}_{3}$ & 464.43 & 142 & 85.6 & 0.63 & 6.04 \\
\hline NBZD-20 & $\mathrm{C}_{27} \mathrm{H}_{27} \mathrm{ClN}_{4} \mathrm{O}_{2}$ & 474.18 & 138 & 67.9 & 0.55 & - \\
\hline NBZD-21 & $\mathrm{C}_{28} \mathrm{H}_{29} \mathrm{ClN}_{6} \mathrm{O}_{3}$ & 532.20 & 142 & 65.2 & 0.76 & 6.59 \\
\hline
\end{tabular}

Rf value: solvent system; chloroform : methanol $1: 1$. 
TABLE 2: Elemental analysis of synthesized compounds.

\begin{tabular}{|c|c|c|c|c|c|c|}
\hline \multirow{2}{*}{ Compound code } & \multicolumn{3}{|c|}{ Calculated value } & \multicolumn{3}{|c|}{ Observed value } \\
\hline & $\mathrm{C}$ & $\mathrm{H}$ & $\mathrm{N}$ & $\mathrm{C}$ & $\mathrm{H}$ & $\mathrm{N}$ \\
\hline NBZD-1 & 80.55 & 9.01 & 10.44 & 80.31 & 8.90 & 10.24 \\
\hline NBZD-3 & 80.96 & 8.05 & 6.99 & 80.56 & 8.00 & 6.79 \\
\hline NBZD-4 & 72.78 & 7.01 & 9.43 & 72.70 & 6.89 & 9.23 \\
\hline NBZD-5 & 74.55 & 7.18 & 6.44 & 74.23 & 7.04 & 6.25 \\
\hline NBZD-6 & 77.70 & 7.11 & 8.24 & 77.56 & 7.01 & 8.16 \\
\hline NBZD-7 & 71.40 & 6.26 & 10.09 & 71.19 & 6.16 & 10.00 \\
\hline NBZD-8 & 72.78 & 6.48 & 7.72 & 72.66 & 6.32 & 7.61 \\
\hline NBZD-9 & 71.88 & 6.74 & 12.33 & 71.34 & 6.66 & 12.21 \\
\hline NBZD-10 & 66.60 & 6.08 & 13.71 & 66.45 & 6.00 & 13.62 \\
\hline NBZD-11 & 67.77 & 6.19 & 11.62 & 67.44 & 6.02 & 11.52 \\
\hline NBZD-12 & 76.55 & 8.57 & 14.88 & 76.25 & 8.34 & 14.54 \\
\hline NBZD-13 & 78.71 & 7.55 & 8.74 & 78.59 & 7.56 & 8.70 \\
\hline NBZD-14 & 69.02 & 6.34 & 11.50 & 68.66 & 6.30 & 11.43 \\
\hline NBZD-15 & 71.07 & 6.53 & 7.89 & 71.00 & 6.45 & 7.68 \\
\hline NBZD-16 & 75.42 & 6.56 & 9.77 & 75.21 & 6.36 & 9.53 \\
\hline NBZD-17 & 68.27 & 5.73 & 11.80 & 68.20 & 5.60 & 11.70 \\
\hline NBZD-18 & 69.83 & 5.83 & 9.05 & 69.70 & 5.78 & 8.90 \\
\hline NBZD-19 & 68.91 & 6.20 & 3.28 & 68.78 & 6.09 & 3.10 \\
\hline NBZD-20 & 63.09 & 5.48 & 15.77 & 63.01 & 5.32 & 15.57 \\
\hline NBZD-21 & 64.37 & 5.59 & 13.40 & 64.30 & 5.40 & 13.28 \\
\hline
\end{tabular}

TABLE 3: Anticonvulsant activity using Isoniazid induced convulsion model.

\begin{tabular}{|c|c|c|c|c|c|}
\hline \multirow{3}{*}{ Compound code } & \multicolumn{3}{|c|}{ Isoniazid induced model } & \multicolumn{2}{|c|}{ Neurotoxicity study } \\
\hline & $0.5 \mathrm{hr}$ & $1 \mathrm{hr}$ & $2 \mathrm{hr}$ & & $4 \mathrm{hr}$ \\
\hline & & & & \multicolumn{2}{|c|}{ Dose: 30 mg/kg } \\
\hline NBZD-1 & $30 \mathrm{mg}$ & $30 \mathrm{mg}$ & Not protected & NN & $\mathrm{NN}$ \\
\hline NBZD-3 & $30 \mathrm{mg}$ & $30 \mathrm{mg}$ & $30 \mathrm{mg}$ & $\mathrm{NN}$ & $\mathrm{NN}$ \\
\hline NBZD-4 & $30 \mathrm{mg}$ & Not protected & Not protected & $\mathrm{NN}$ & $\mathrm{NN}$ \\
\hline NBZD-5 & $30 \mathrm{mg}$ & Not protected & Not protected & $\mathrm{NN}$ & $\mathrm{NN}$ \\
\hline NBZD-6 & $30 \mathrm{mg}$ & $30 \mathrm{mg}$ & Not protected & $\mathrm{NN}$ & $\mathrm{NN}$ \\
\hline NBZD-7 & $30 \mathrm{mg}$ & Not protected & Not protected & $\mathrm{NN}$ & $\mathrm{NN}$ \\
\hline NBZD-8 & $30 \mathrm{mg}$ & $30 \mathrm{mg}$ & $30 \mathrm{mg}$ & $\mathrm{NN}$ & $\mathrm{NN}$ \\
\hline NBZD-9 & $30 \mathrm{mg}$ & Not protected & Not protected & $\mathrm{NN}$ & $\mathrm{NN}$ \\
\hline NBZD-10 & $30 \mathrm{mg}$ & $30 \mathrm{mg}$ & Not protected & $\mathrm{NN}$ & $\mathrm{NN}$ \\
\hline NBZD-11 & $30 \mathrm{mg}$ & $30 \mathrm{mg}$ & Not protected & $\mathrm{NN}$ & $\mathrm{NN}$ \\
\hline Control & - & - & - & & \\
\hline
\end{tabular}

Symbol (NN) indicates no neurotoxicity at $30 \mathrm{mg} / \mathrm{kg}$ b.w.

$\left.2 \mathrm{H},-\mathrm{NHCH}_{2}\right), \mathrm{CH}\left(\mathrm{m}, 6.4-7.0,4 \mathrm{H}\right.$, phenyl), $\mathrm{CH}_{2}(\mathrm{~m}$, $1.2-1.5,18 \mathrm{H}$, cyclohexane $), \mathrm{CH}(\mathrm{s}, 2.5,1 \mathrm{H}$, diazepine ring).

IR (KBr): $\mathrm{C}=\mathrm{O}\left(1700 \mathrm{~cm}^{-1}, \mathrm{str}\right), \mathrm{CH}\left(\mathrm{Ar}, 3180 \mathrm{~cm}^{-1}\right.$, str), $\mathrm{CH}\left(\mathrm{Ar}, 810 \mathrm{~cm}^{-1}\right.$, bend $), \mathrm{C}=\mathrm{N}\left(1618 \mathrm{~cm}^{-1}\right.$, Str $)$, $\mathrm{CH}_{2}\left(1490 \mathrm{~cm}^{-1}\right.$, str $), \mathrm{C}-\mathrm{C}\left(\mathrm{Ar}, 1600 \mathrm{~cm}^{-1}\right), \mathrm{C}=\mathrm{C}(\mathrm{Ar}$, $\left.1410,1500,1580 \mathrm{~cm}^{-1}\right)$.
(3) 1-(4-Nitrophenyl)-3-(10-spirocyclohexane-1,2,3,9, 10,10a-hexahydrobenzo[b]cyclohexane $[\mathrm{e}][1,4]$ diazepine-1-yl) propan-1-one (NBZD-4).

${ }^{1} \mathrm{H}$ NMR (300 MHz, $\left.\delta\right): \mathrm{CH}(\mathrm{m}, 8.1-8.2$, 4H, p-nitroacetophenone), $\mathrm{CH}_{2}\left(\mathrm{~s}, 2.7,2 \mathrm{H},-\mathrm{COCH}_{2}\right), \mathrm{CH}_{2}(\mathrm{~s}$, $\left.3.5,2 \mathrm{H},-\mathrm{NHCH}_{2}\right), \mathrm{CH}(\mathrm{m}, 6.4-7.0,4 \mathrm{H}$, phenyl), 
TABLE 4: Anticonvulsant activity using thiosemicarbazide induced convulsion model.

\begin{tabular}{|c|c|c|c|c|c|}
\hline \multirow{3}{*}{ Compound code } & \multicolumn{3}{|c|}{ Thiosemicarbazide induced model } & \multicolumn{2}{|c|}{ Neurotoxicity study } \\
\hline & $0.5 \mathrm{hr}$ & $1 \mathrm{hr}$ & $2 \mathrm{hr}$ & & $4 \mathrm{hr}$ \\
\hline & & & & \multicolumn{2}{|c|}{ Dose: 30 mg/kg } \\
\hline NBZD-12 & $30 \mathrm{mg}$ & $30 \mathrm{mg}$ & Not protected & $\mathrm{NN}$ & $\mathrm{NN}$ \\
\hline NBZD-13 & $30 \mathrm{mg}$ & $30 \mathrm{mg}$ & $30 \mathrm{mg}$ & $\mathrm{NN}$ & $\mathrm{NN}$ \\
\hline NBZD-14 & $30 \mathrm{mg}$ & Not protected & Not protected & NN & NN \\
\hline NBZD-15 & $30 \mathrm{mg}$ & Not protected & Not protected & $\mathrm{NN}$ & $\mathrm{NN}$ \\
\hline NBZD-16 & $30 \mathrm{mg}$ & $30 \mathrm{mg}$ & Not protected & $\mathrm{NN}$ & $\mathrm{NN}$ \\
\hline NBZD-17 & $30 \mathrm{mg}$ & Not protected & Not protected & $\mathrm{NN}$ & $\mathrm{NN}$ \\
\hline NBZD-18 & $30 \mathrm{mg}$ & $30 \mathrm{mg}$ & $30 \mathrm{mg}$ & NN & NN \\
\hline NBZD-19 & $30 \mathrm{mg}$ & Not protected & Not protected & NN & NN \\
\hline NBZD-20 & $30 \mathrm{mg}$ & Not protected & Not protected & NN & NN \\
\hline NBZD-21 & $30 \mathrm{mg}$ & $30 \mathrm{mg}$ & Not protected & NN & $\mathrm{NN}$ \\
\hline Control & - & - & - & & \\
\hline
\end{tabular}

Symbol (NN) indicates no neurotoxicity $30 \mathrm{mg} / \mathrm{kg}$ of b.w.

TABLE 5: Sedative activity of synthesized compound.

\begin{tabular}{lc}
\hline Compounds code & $\begin{array}{c}\text { Sleeping time (mean } \pm \text { SEM) } \\
(\mathrm{min})\end{array}$ \\
\hline NBZD-1 & $120 \pm 9.00^{* *}$ \\
NBZD-3 & $138 \pm 10.53^{* *}$ \\
NBZD-4 & $140 \pm 11.92^{* *}$ \\
NBZD-7 & $141 \pm 11.21^{* *}$ \\
NBZD-8 & $68 \pm 12.6 \mathrm{NS}$ \\
NBZD-10 & $63 \pm 9.05 \mathrm{NS}$ \\
NBZD-11 & $148 \pm 12.15^{* *}$ \\
NBZD-12 & $124 \pm 10.12^{* *}$ \\
NBZD-14 & $110 \pm 11.41^{* *}$ \\
NBZD-15 & $100 \pm 10.98^{* *}$ \\
NBZD-17 & $148 \pm 11.54^{* *}$ \\
NBZD-18 & $76 \pm 10.26 \mathrm{NS}$ \\
NBZD-20 & $112 \pm 9.62^{* *}$ \\
NBZD-21 & $157 \pm 12.09^{* *}$ \\
Phenobarbitone (control ) & $56 \pm 11.47$ \\
\hline
\end{tabular}

Values represent the mean \pm SEM of six animals for each group.

*Significant at $P<0.05$ and ${ }^{* *}$ significant at $P<0.01$ (Dunnett's test).

Test drug $(30 \mathrm{mg} / \mathrm{kg})$ and Phenobarbitone $(40 \mathrm{mg} / \mathrm{kg})$.

NS denotes not significant at $P<0.01$ (student's $t$-test).

$\mathrm{NH}(\mathrm{s}, 4.0,1 \mathrm{H}),, \mathrm{CH}_{2}(\mathrm{~m}, 1.22-1.59,18 \mathrm{H}$, cyclohexane), $\mathrm{CH}(\mathrm{s}, 2.7,1 \mathrm{H}$, diazepine ring).

IR ( $\mathrm{KBr}): \mathrm{C}=\mathrm{O}\left(1710 \mathrm{~cm}^{-1}\right.$, str), $\mathrm{CH}\left(\mathrm{Ar}, 3150 \mathrm{~cm}^{-1}\right.$, str), $\mathrm{CH}\left(\mathrm{Ar}, 800 \mathrm{~cm}^{-1}\right.$, bend $), \mathrm{C}=\mathrm{N}\left(1658 \mathrm{~cm}^{-1}\right.$, Str), $\mathrm{CH}_{2}\left(1490 \mathrm{~cm}^{-1}, \mathrm{str}\right), \mathrm{C}-\mathrm{C}\left(\mathrm{Ar}, 1610 \mathrm{~cm}^{-1}\right), \mathrm{C}=\mathrm{C}(\mathrm{Ar}$, $\left.1410,1560,1580 \mathrm{~cm}^{-1}\right), \mathrm{N}-\mathrm{O}\left(1350 \mathrm{~cm}^{-1}\right.$, str $)$.

(4) (4-Chloro-phenyl)-[1-chlorophenyl-3-10-spirocyclohexane-1,2,3,9,10,10a-hexahydrobenzo[b]cyclohexane[e] [1,4]diazepine-1yl)-propylidene]-amine

(NBZD-8).
${ }^{1} \mathrm{H}$ NMR $(300 \mathrm{MHz}, \delta): \mathrm{CH}(\mathrm{m}, 7.30-7.5,4 \mathrm{H}, \mathrm{p}-$ chloroacetophenone $), \mathrm{CH}(\mathrm{m}, 7.2-7.3,4 \mathrm{H}$, p-chloroaniline), $\mathrm{CH}_{2}\left(\mathrm{~s}, 1.6,2 \mathrm{H},-\mathrm{COCH}_{2}\right), \mathrm{CH}_{2}(\mathrm{~s}, 3.4$, $\left.2 \mathrm{H},-\mathrm{NHCH}_{2}\right), \mathrm{CH}\left(\mathrm{m}, 6.6-7.1,4 \mathrm{H}\right.$, phenyl), $\mathrm{CH}_{2}(\mathrm{~m}$, $1.3-1.5,18 \mathrm{H}$, cyclohexane $), \mathrm{CH}(\mathrm{s}, 2.7,1 \mathrm{H}$, diazepine ring).

IR (KBr): $\mathrm{C}=\mathrm{N}\left(1569 \mathrm{~cm}^{-1}, \mathrm{str}\right), \mathrm{C}-\mathrm{Cl}\left(727 \mathrm{~cm}^{-1}, \mathrm{str}\right)$, $\mathrm{C}-\mathrm{Cl}\left(760 \mathrm{~cm}^{-1}\right), \mathrm{C}-\mathrm{H}\left(2975 \mathrm{~cm}^{-1}\right.$, str assym), CH $\left(1383.9 \mathrm{~cm}^{-1}\right.$, def sym.), C-H(Ar, $3072 \mathrm{~cm}^{-1}$, str), $\mathrm{CH}\left(\mathrm{Ar}, 3150 \mathrm{~cm}^{-1}\right.$, str), $\mathrm{CH}\left(\mathrm{Ar}, 860 \mathrm{~cm}^{-1}\right.$, bend), $\mathrm{C}=\mathrm{N}\left(1678 \mathrm{~cm}^{-1}\right.$, Str) $\mathrm{CH}_{2}\left(1490 \mathrm{~cm}^{-1}, \mathrm{str}\right), \mathrm{C}-\mathrm{C}(\mathrm{Ar}$, $\left.1600 \mathrm{~cm}^{-1}\right), \mathrm{C}=\mathrm{C}\left(\mathrm{Ar}, 1410,1500,1580 \mathrm{~cm}^{-1}\right)$.

(5) (4-Chlorophenylhyrazinecarboxamide) [1-nitrophenyl-3-(10-spirocyclohexane-1,2,3,9,10,10a-hexahydrobenzo[b]cyclohexane $[\mathrm{e}][1,4]$ diazepine-1-yl)propylidene]-amine (NBZD-10).

${ }^{1} \mathrm{H}$ NMR $(300 \mathrm{MHz}, \delta): \mathrm{CH}(\mathrm{m}, 7.9-8.2,4 \mathrm{H}, \mathrm{p}-$ nitroacetophenone), $\mathrm{NH}(\mathrm{s}, 7.0,1 \mathrm{H},=\mathrm{NNH}$, p-chlorophenylsemicarbazide), $\mathrm{NH}\left(\mathrm{s}, 6.0,1 \mathrm{H},-\mathrm{NHC}_{6} \mathrm{H}_{4}\right.$ $\mathrm{Cl}$, p-chlorophenylsemicarbazide), $\mathrm{CH}$ ( $\mathrm{m}, 7.2-7.6$, $4 \mathrm{H}$, p-chlorophenylsemicarbazide), $\mathrm{CH}$ (m, 6.6-7.1, $4 \mathrm{H}$, phenyl $), \mathrm{CH}_{2}(\mathrm{~m}, 1.3-1.5,18 \mathrm{H}$, cyclohexane), $\mathrm{CH}(\mathrm{s}, 2.3,1 \mathrm{H}$, diazepine ring $), \mathrm{CH}_{2}(\mathrm{~s}, 1.6,2 \mathrm{H},-$ $\left.\mathrm{COCH}_{2}\right), \mathrm{CH}_{2}\left(\mathrm{~s}, 3.4,2 \mathrm{H},-\mathrm{NHCH}_{2}\right)$.

IR ( $\mathrm{KBr}): \mathrm{C}=\mathrm{N}\left(1569 \mathrm{~cm}^{-1}, \mathrm{str}\right), \mathrm{C}-\mathrm{Cl}\left(728 \mathrm{~cm}^{-1}\right.$, str), $\mathrm{C}-\mathrm{H}\left(2970 \mathrm{~cm}^{-1}\right.$, str assym $), \mathrm{CH}\left(1353.9 \mathrm{~cm}^{-1}\right.$, def sym.), $\mathrm{C}-\mathrm{H}\left(\mathrm{Ar}, 3062 \mathrm{~cm}^{-1}\right.$, str $), \mathrm{CH}\left(\mathrm{Ar}, 3180 \mathrm{~cm}^{-1}\right.$, str), $\mathrm{CH}\left(\mathrm{Ar}, 810 \mathrm{~cm}^{-1}\right.$, bend $), \mathrm{C}=\mathrm{N}\left(1638 \mathrm{~cm}^{-1}\right.$, Str) $\mathrm{CH}_{2}\left(1490 \mathrm{~cm}^{-1}\right.$, str $), \mathrm{C}-\mathrm{C}\left(\mathrm{Ar}, 1680 \mathrm{~cm}^{-1}\right), \mathrm{C}=\mathrm{C}(\mathrm{Ar}$, $\left.1410,1500,1580 \mathrm{~cm}^{-1}\right), \mathrm{NO}\left(1380 \mathrm{~cm}^{-1}\right.$, str $)$.

(6) (4-Chlorophenylhyrazinecarboxamide) [1-nitrophenyl-3-(10-spirocyclohexane-1,2,3,9,10,10a-hexahydrobenzo[b]cyclohexane $[\mathrm{e}][1,4]$ diazepine-1-yl)propylidene]-amine (NBZD-11):

${ }^{1} \mathrm{H}$ NMR $(300 \mathrm{MHz}, \delta): \mathrm{CH}(\mathrm{m}, 7.6-7.7,4 \mathrm{H}, \mathrm{p}-$ chloroacetophenone $), \quad \mathrm{NH}(\mathrm{s}, 9.0,1 \mathrm{H},=\mathrm{NNH}$, 
p-chlorophenylsemicarbazide), $\mathrm{NH}(\mathrm{s}, \quad 6.0,1 \mathrm{H}$, - $\mathrm{NHC}_{6} \mathrm{H}_{4} \mathrm{Cl}$, p-chlorophenylsemicarbazide), $\mathrm{CH}(\mathrm{m}$, 7.2-7.6,4H, p-chlorophenylsemicarbazide), $\mathrm{CH}$ (m, 6.6-7.1, 4H, phenyl), $\mathrm{CH}_{2}(\mathrm{~m}, 1.3-1.6,18 \mathrm{H}$, cyclohexane), $\mathrm{CH}\left(\mathrm{s}, 2.7,1 \mathrm{H}\right.$, diazepine ring), $\mathrm{CH}_{2}(\mathrm{~s}, 1.6$, $\left.2 \mathrm{H},-\mathrm{COCH}_{2}\right), \mathrm{CH}_{2}\left(\mathrm{~s}, 3.4,2 \mathrm{H},-\mathrm{NHCH}_{2}\right)$.

IR (KBr): $\mathrm{C}=\mathrm{N}\left(1559 \mathrm{~cm}^{-1}\right.$, str $), \mathrm{C}-\mathrm{Cl}\left(787 \mathrm{~cm}^{-1}\right.$, str), C-Cl( $\left.769 \mathrm{~cm}^{-1}\right), \mathrm{C}-\mathrm{H}\left(2975 \mathrm{~cm}^{-1}\right.$, str assym), $\mathrm{CH}\left(1353.9 \mathrm{~cm}^{-1}\right.$, def sym. $), \mathrm{C}-\mathrm{H}\left(\mathrm{Ar}, 3062 \mathrm{~cm}^{-1}\right.$, str), $\mathrm{CH}\left(\mathrm{Ar}, 3180 \mathrm{~cm}^{-1}\right.$, str $), \mathrm{CH}\left(\mathrm{Ar}, 880 \mathrm{~cm}^{-1}\right.$, bend $), \quad \mathrm{C}=\mathrm{N}\left(1638 \mathrm{~cm}^{-1}, \quad \mathrm{Str}\right) \quad \mathrm{CH}_{2}\left(1490 \mathrm{~cm}^{-1}\right.$, str), $\mathrm{C}-\mathrm{C}\left(\mathrm{Ar}, 1600 \mathrm{~cm}^{-1}\right), \mathrm{C}=\mathrm{C}(\mathrm{Ar}, 1410,1500$, $\left.1580 \mathrm{~cm}^{-1}\right)$.

(7) (1-Phenyl-3-(2,2,4-trimethyl-2,3-dihydrobenzo[b] $[1,4]$ diazepin-1-yl)-propan-1-one) (NBZD-13).

${ }^{1} \mathrm{H}$ NMR $(300 \mathrm{MHz}, \delta): \mathrm{CH}(\mathrm{m}, 7.3-7.8,5 \mathrm{H}$, Acetophenone), $\mathrm{CH}_{2}\left(\mathrm{~s}, 2.7,2 \mathrm{H},-\mathrm{COCH}_{2}\right), \mathrm{CH}_{2}$ (s, $\left.3.5,2 \mathrm{H},-\mathrm{NHCH}_{2}\right), \mathrm{CH}(\mathrm{m}, 6.6-7.1,4 \mathrm{H}$, phenyl), $2 \mathrm{xCH}_{3}(\mathrm{~s}, 1.28,6 \mathrm{H}), \mathrm{CH}_{3}(\mathrm{~s}, 0.9,3 \mathrm{H}), \mathrm{CH}_{2}(\mathrm{~s}, 2.5,2 \mathrm{H}$, diazepine ring).

IR (KBr): $\mathrm{C}=\mathrm{O}\left(1700 \mathrm{~cm}^{-1}\right.$, str $), \mathrm{NH}\left(\mathrm{Ar}, 3230 \mathrm{~cm}^{-1}\right.$, str), $\mathrm{CH}\left(\mathrm{Ar}, 3180 \mathrm{~cm}^{-1}\right.$, str $), \mathrm{CH}\left(\mathrm{Ar}, 800 \mathrm{~cm}^{-1}\right.$, bend $), \quad \mathrm{C}=\mathrm{N}\left(1618 \mathrm{~cm}^{-1}, \quad \mathrm{Str}\right), \quad \mathrm{CH}_{3}\left(2980 \mathrm{~cm}^{-1}\right.$, str $), \quad C-C\left(A r, 1610 \mathrm{~cm}^{-1}\right), \quad C=C(A r, 1410,1500$, $\left.1580 \mathrm{~cm}^{-1}\right)$.

(8) 1-(4-Chloro-phenyl)-3-(2,2,4-trimethyl-2,3-dihydrobenzo[b][1,4] diazepin-1-yl)-propan-1-one (NBZD-15).

${ }^{1} \mathrm{H}$ NMR $(300 \mathrm{MHz}, \delta): \mathrm{CH}(\mathrm{m}, 7.3-7.8,4 \mathrm{H}, \mathrm{p}-$ chloroacetophenone), $\mathrm{CH}_{2}\left(\mathrm{~s}, 2.78,2 \mathrm{H},-\mathrm{COCH}_{2}\right)$, $\mathrm{CH}_{2}\left(\mathrm{~s}, 3.5,2 \mathrm{H},-\mathrm{NHCH}_{2}\right), \mathrm{CH}(\mathrm{m}, 6.6-7.1,4 \mathrm{H}$, phenyl), $2 \mathrm{xCH}_{3}(\mathrm{~s}, 1.2,6 \mathrm{H}), \mathrm{CH}_{3}(\mathrm{~s}, 0.9,3 \mathrm{H}), \mathrm{CH}_{2}$ (s, 2.4, 2H, Diazepine ring).

IR (KBr): $\mathrm{C}=\mathrm{O}\left(1710 \mathrm{~cm}^{-1}\right.$, str $), \mathrm{NH}\left(\mathrm{Ar}, 3030 \mathrm{~cm}^{-1}\right.$, str), $\mathrm{CH}\left(\mathrm{Ar}, 3280 \mathrm{~cm}^{-1}\right.$, str $), \mathrm{CH}\left(\mathrm{Ar}, 800 \mathrm{~cm}^{-1}\right.$, bend $), \quad \mathrm{C}=\mathrm{N}\left(1618 \mathrm{~cm}^{-1}, \quad \mathrm{Str}\right), \quad \mathrm{CH}_{3}\left(2990 \mathrm{~cm}^{-1}\right.$, str), C-C $\left(\mathrm{Ar}, 1600 \mathrm{~cm}^{-1}\right), \mathrm{C}=\mathrm{C}(\mathrm{Ar}, 1410,1500$, $\left.1580 \mathrm{~cm}^{-1}\right), \mathrm{C}-\mathrm{Cl}\left(760 \mathrm{~cm}^{-1}\right)$.

(9) (4-Chloro-phenyl)-[1-(4-nitrophenyl)-3-(2,2,4-trimethyl-2,3-dihydro-benzo[b][1,4] dizepine-1-yl)propylidene (NBZD-17).

${ }^{1} \mathrm{H}$ NMR $(300 \mathrm{MHz}, \delta): \mathrm{CH}(\mathrm{m}, 7.8-8.2,4 \mathrm{H}, \mathrm{p}-$ nitroacetophenone $), \mathrm{CH}(\mathrm{m}, 7.2-7.3,4 \mathrm{H}, \mathrm{p}$-chloroaniline), $\mathrm{CH}_{2}\left(\mathrm{~s}, 1.6,2 \mathrm{H},-\mathrm{N}=\mathrm{C}-\mathrm{CH}_{2}\right), \mathrm{CH}_{2}(\mathrm{~s}$, $\left.3.4,2 \mathrm{H},-\mathrm{NHCH}_{2}\right), \mathrm{CH}(\mathrm{m}, 6.6-7.1,4 \mathrm{H}$, phenyl), $2 \mathrm{xCH}_{3}(\mathrm{~s}, 1.2,6 \mathrm{H}), \mathrm{CH}_{3}(\mathrm{~s}, 0.9,3 \mathrm{H}), \mathrm{CH}_{2}(\mathrm{~s}, 2.5,2 \mathrm{H}$, diazepine ring).

IR (KBr): C=N $\left(1599 \mathrm{~cm}^{-1}\right.$, str $), \mathrm{C}-\mathrm{Cl}\left(728 \mathrm{~cm}^{-1}\right.$, str $)$, $\mathrm{C}-\mathrm{H}\left(2985 \mathrm{~cm}^{-1}\right.$, str assym), $\mathrm{CH}\left(1353.9 \mathrm{~cm}^{-1}\right.$, def sym. ), C-H(Ar, $3062 \mathrm{~cm}^{-1}$, str $), \mathrm{CH}\left(\mathrm{Ar}, 3180 \mathrm{~cm}^{-1}\right.$, str), $\mathrm{CH}\left(\mathrm{Ar}, 800 \mathrm{~cm}^{-1}\right.$, bend $), \mathrm{C}=\mathrm{N}\left(1618 \mathrm{~cm}^{-1}, \mathrm{Str}\right)$, $\mathrm{CH}_{3}\left(2990 \mathrm{~cm}^{-1}\right.$, str $), \mathrm{C}-\mathrm{C}\left(\mathrm{Ar}, 1600 \mathrm{~cm}^{-1}\right), \mathrm{C}=\mathrm{C}(\mathrm{Ar}$, $\left.1410,1500,1580 \mathrm{~cm}^{-1}\right), \mathrm{NO}\left(1350 \mathrm{~cm}^{-1}\right.$, str $)$

(10) 4-Chlorophenylhyrazinecarboxamide) [1-nitrophenyl-3-(2,2,4-trimethyl-2,3-dihydrobenzo[b][1,4] diazepine-1-yl)-propylidene]-amine (NBZD-20).
${ }^{1} \mathrm{H} \quad \mathrm{NMR} \quad(300 \mathrm{MHz}, \delta): \mathrm{CH}(\mathrm{m}, \quad 7.9-8.2, \quad 4 \mathrm{H}$, p-nitroacetophenone), $\mathrm{NH}(\mathrm{s}, 9.0,1 \mathrm{H},=\mathrm{NNH}$, p-chlorophenylsemicarbazide), $\mathrm{NH}(\mathrm{s}, 6.0,1 \mathrm{H}$, $-\mathrm{NHC}_{6} \mathrm{H}_{4} \mathrm{Cl}$, p-chlorophenylsemicarbazide $), \mathrm{CH}(\mathrm{m}$, 7.2-7.5,4H, p-chlorophenylsemicarbazide), $\mathrm{CH}_{2}$ (s, $\left.1.6,2 \mathrm{H},-\mathrm{N}=\mathrm{C}-\mathrm{CH}_{2}\right), \mathrm{CH}_{2}\left(\mathrm{~s}, 3.4,2 \mathrm{H},-\mathrm{NHCH}_{2}\right)$, $\mathrm{CH}(\mathrm{m}, 6.6-7.1,4 \mathrm{H}$, phenyl $), 2 \mathrm{xCH}_{3}(\mathrm{~s}, 1.28,6 \mathrm{H})$, $\mathrm{CH}_{3}(\mathrm{~s}, 0.9,3 \mathrm{H}), \mathrm{CH}_{2}(\mathrm{~s}, 2.5,2 \mathrm{H}$, diazepine ring).

IR (KBr): $\mathrm{C}=\mathrm{N}\left(1570 \mathrm{~cm}^{-1}, \mathrm{str}\right), \mathrm{C}-\mathrm{Cl}\left(730 \mathrm{~cm}^{-1}, \mathrm{str}\right)$, $\mathrm{C}-\mathrm{H}\left(2975 \mathrm{~cm}^{-1}\right.$, str assym $), \mathrm{CH}\left(1353.9 \mathrm{~cm}^{-1}\right.$, def sym. $), \mathrm{C}-\mathrm{H}\left(\mathrm{Ar}, 3062 \mathrm{~cm}^{-1}\right.$, str $), \mathrm{CH}\left(\mathrm{Ar}, 3180 \mathrm{~cm}^{-1}\right.$, str), $\mathrm{CH}\left(\mathrm{Ar}, 800 \mathrm{~cm}^{-1}\right.$, bend $), \mathrm{C}=\mathrm{N}\left(1618 \mathrm{~cm}^{-1}, \mathrm{Str}\right)$, $\mathrm{CH}_{3}\left(2990 \mathrm{~cm}^{-1}\right.$, str $), \mathrm{C}-\mathrm{C}\left(\mathrm{Ar}, 1600 \mathrm{~cm}^{-1}\right), \mathrm{C}=\mathrm{C}(\mathrm{Ar}$, $\left.1410,1500,1580 \mathrm{~cm}^{-1}\right), \mathrm{NO}\left(1350 \mathrm{~cm}^{-1}\right.$, str $)$.

4.4. Anticonvulsant Activity Using Chemical Induced Method. See Tables 3 and 4 .

\subsection{Sedative Activity. See Table 5.}

\section{Conclusion}

All the synthesized derivatives were evaluated at the dose of $30 \mathrm{mg} / \mathrm{kg}$ b.w for anticonvulsant activity by isoniazid induced convulsion model and the compounds NBZD3 and NBZD-8 were found to be most active among all compounds. Among all the synthesized derivatives, compounds NBZD-13, and NBZD-17 were found to be most active among all compounds using thiosemicarbazide induced model. Activity of the drugs interfering with motor coordination was checked by the rotarod test. None of the synthesized compounds were found to be neurotoxic at a dose of $30 \mathrm{mg} / \mathrm{kg}$ b.w among all the tested compounds. The compounds NBZD-1, NBZD-3, NBZD-4, NBZD-7, NBZD11, NBZD-12, NBZD-14, NBZD-15, NBZD-17, NBZD-20, NBZD-21 were found to cause sedation. Although NBZD8, NBZD-10, and NBZD-18 are the compounds which had shown good anticonvulsant activity and have an advantage over that, they were not sedative.

Sulfated zirconia is one of the important agent that has attracted much attention recently because of its superacidity, nontoxicity, and low cost. Sulfated zirconia catalyzes many reactions under very mild condition in vapor as well as liquid phase.

\section{Structure Activity Relationship}

\subsection{Anticonvulsant Scheme 9}

(1) Highly active: NBZD-3, NBZD-8, NBZD-13, and NBZD-18.

(2) Moderately active: NBZD-1, NBZD-6, NBZD-10, NBZD-11, NBZD-12, and NBZD-16, NBZD-21.

(3) Less active: NBZD-4, NBZD-7, NBZD-9, NBZD-14, NBZD-15, NBZD-17, NBZD-19, and NBZD- 20.

Most functional subtypes of the $\mathrm{GABA}_{\mathrm{A}}$ receptor contain $\alpha, \beta$, subunits, with the different benzodiazepine binding 
site ligands. BZ-binding site ligands act through mechanisms which modulate the inhibiting effects of GABA.

(1) In the basic structure of benzodiazepine, early SAR studies indicated that the seven-membered imino ring $\mathrm{B}$ was essential for its affinity towards the BZbinding site.

(2) 4-5 Carbimino double bond has also been shown to substantially contribute to the binding affinity of compound. Saturation leads to complete less of activity. It acts as a two-electron donor site.

(3) The primary chemical moieties of the compounds which contribute to high receptor binding affinity are restricted to positions $7,2,1$.

(a) Position 2 is the most effective place. Presence of an electrophilic and bulky substituent at position 2 results in strong increase in receptor binding affinity of the corresponding compounds.

(b) Compounds NBZD-3 and NBZD-8 had shown good anticonvulsant activity as they have cyclohexane ring at position 2 .

(4) Molar refractivity is the most important parameter at position 1, suggesting that the molecular size of the substituent needs to be restricted at position 1 for effective ligand binding. Compounds NBZD-1 and NBZD-8 have less substituent as compared to other and hence more active.

(5) Compounds NBZD-6, NBZD-10, and NBZD-11 were found to be moderately active anticonvulsant action, hence this shows that chloro-substituted derivatives are rather good anticonvulsant agent as compared to nitro-substituted derivatives.

(6) Among these synthesized compounds which have methyl groups at the 2nd and 4th position, NBZD13, and NBZD-18 were found to be most active. Hence it shows that good activity compounds are preferred with less substitution the at 1st position.

\section{Acknowledgments}

The authors are thankful to Central Drug Research Institute, Lucknow, India. They express their thanks to the Head and the Department of Pharmaceutical Sciences, Saroj Institute of Technology and Management, Lucknow, India, for their assistance during the course of study.

\section{References}

[1] C. Page, C. Michael, M. Sutter, M. Walker, and B. Hoffman, Integrated Pharmacology, Mosby, 2nd edition, 2005.

[2] G. A. Archer and L. H. Sternbach, "The chemistry of benzodiazepines," Chemical Reviews, vol. 68, no. 6, pp. 747784, 1968.

[3] R. H. Smith Jr., W. L. Jorgensen, J. Tirado-Rives et al., "Prediction of binding affinities for TIBO inhibitors of HIV-1 reverse transcriptase using Monte Carlo simulations in a linear response method," Journal of Medicinal Chemistry, vol. 41, no. 26, pp. 5272-5286, 1998.

[4] M. A. Rogowaski and R. J. Porter, "Antiepileptic drugs: pharmacological mechanisms and clinical efficacy with consideration of promising developmental stage compounds," Pharmacological Reviews, vol. 42, no. 3, pp. 223-286, 1990.

[5] R. Fisher and D. Blum, "Clobazam, oxcarbazepine, tiagabine, topiramate, and other new antiepileptic drugs," Epilepsia, vol. 36, no. 2, pp. S105-S114, 1995.

[6] L. Steru, R. Chermat, and B. Millet, "Comparative study in mice of ten 1,4-benzodiazepines and of clobazam: anticonvulsant, anxiolytic, sedative, and myorelaxant effects," Epilepsia, vol. 27, no. 1, pp. S14-S17, 1986.

[7] A. K. Shenoy, J. T. Miyahara, E. A. Swinyard, and H. J. Kuperberg, "Comparative anticonvulsant activity and neurotoxicity of clobazam, diazepam, phenobarbital, and valproate in mice and rats," Epilepsia, vol. 23, no. 4, pp. 399-408, 1982.

[8] K. P. Jadhav and D. B. Ingled, "Synthis of 2,4-diary12,3-dihyrdo-1,5-benzothiazeoines and thier 1,1-dioxides as antibacterial agents," Indian Journal of Chemistry, vol. B22, p. $180,1983$.

[9] R. J. Reddy, D. Ashok, and P. N. Sharma, "Synthesis of 4.6-bis(2'-substitutcri-2-3'-dihydro-1.5- benzothiazepin$4^{\prime}$-yl)resorcinols as potential antifeedants," Indian Journal of Chemistry B, vol. 32, pp. 404-406, 1993.

[10] K. Satyanarayana and M. N. A. Rao, "Synthesis of 3[4-[2t 3-dihydro-2-(substituted aryl)-1,5- benzothiazcpin-4yl]phcnyl]sydnones as potential antiinflammatory agents," Indian Journal of Pharmaceutical Sciences, vol. 55, pp. 230-233, 1993.

[11] G. Dessarro, A. Chimirri, A. Dessaro, and R. Gitto, Chemical Abstracts, vol. 106, p. 11222g, 1995.

[12] J. K. Landquist, "Application as pharmaceuticals," in Comprehensive Heterocyclic Chemistry, A. R. Kalritzky and C. W. Rees, Eds., vol. 1, pp. 143-183, Peramon, Oxford, UK, 1984.

[13] L. H. Sternbach, "The benzodiazepine story," Journal of Medicinal Chemistry, vol. 22, no. 1, pp. 1-7, 1979.

[14] P. Danneberg and K. H. Weber, "Chemical structure and biological activity of the diazepines," British Journal of Clinical Pharmacology, vol. 16, no. 2, pp. 231S-243S, 1983.

[15] A. Walser and R. I. Fryer, Chemical Abstracts, vol. 89, p. 59906, 1978.

[16] T. Hashiyama, H. Inoue, M. Takeda, S. Murtates, and T. Nagao, "Reactions of 3-phenylglycidic esters. V. Reaction of methyl 3-(4-methoxyphenyl) glycidate with 2-nitroaniline and synthesis of 1,5-benzodiazepine derivatives," Chemical \& Pharmaceutical Bulletin, vol. 33, pp. 2348-2358, 1985.

[17] J. K. Chakrabarti, L. Horsman, T. M. Hotten, I. A. Pullar, D. E. Tupper, and F. C. Wright, "4-Piperazinyl-10H-thieno[2,3b] $[1,5]$ benzodiazepines as potential neuroleptics," Journal of Medicinal Chemistry, vol. 23, no. 8, pp. 878-884, 1980.

[18] S. Caccia, G. Grossi, C. Fracasso et al., "Acid-catalysed hydrolysis and benzodiazepine-like properties of 5-(dialkylamino)- and 5-(alkylthio)-substituted 8-chloro-6-phenyl$6 \mathrm{H}$-[1,2,4] triazolo[4,3-a] [1,5]benzodiazepines in mice," Journal of Pharmacy and Pharmacology, vol. 50, no. 7, pp. 723-728, 1998.

[19] A. Bauer, K. H. Weber, and P. Danneberg, Chemical Abstracts, vol. 82, p. 4331, 1975.

[20] R. Unny, P. Joshi, M. P. Dobhal, and Y. C. Joshi, "Synthesis of 1,3-diketone and its reaction with different $N$-nucleophiles (part II)," Heterocyclic Communications, vol. 9, no. 2, pp. 171174, 2003. 
[21] N. Fokialakis, P. Magiatis, I. Chinou, S. Mitaku, and F. Tillequin, "Megistoquinones I and II, two quinoline alkaloids with antibacterial activity from the bark of Sarcomelicope megistophylla," Chemical and Pharmaceutical Bulletin, vol. 50, no. 3, pp. 413-414, 2002.

[22] R. Pili, Proceedings of the American Association for Cancer Research, vol. 43, p. 5363, 2002.

[23] N. K. Raju, T. S. Seniappan, S. Thangaraju, and S. M. Palathurai, Heterocycles, vol. 52, no. 2, p. 357, 2000. 


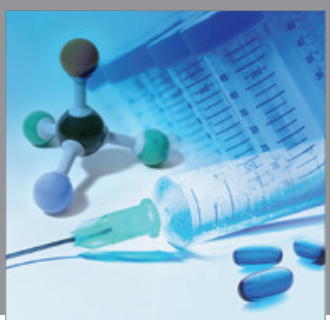

International Journal of

Medicinal Chemistry

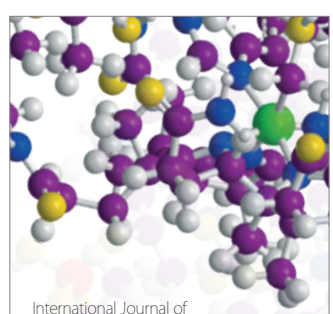

Carbohydrate Chemistry

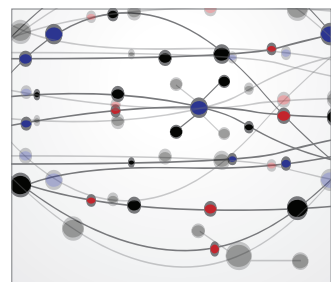

The Scientific World Journal
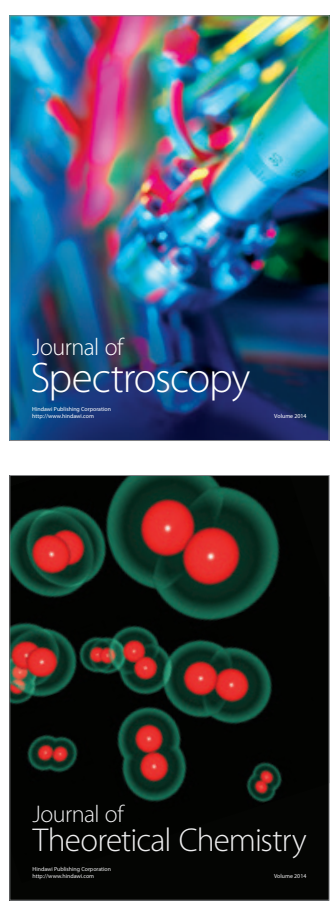
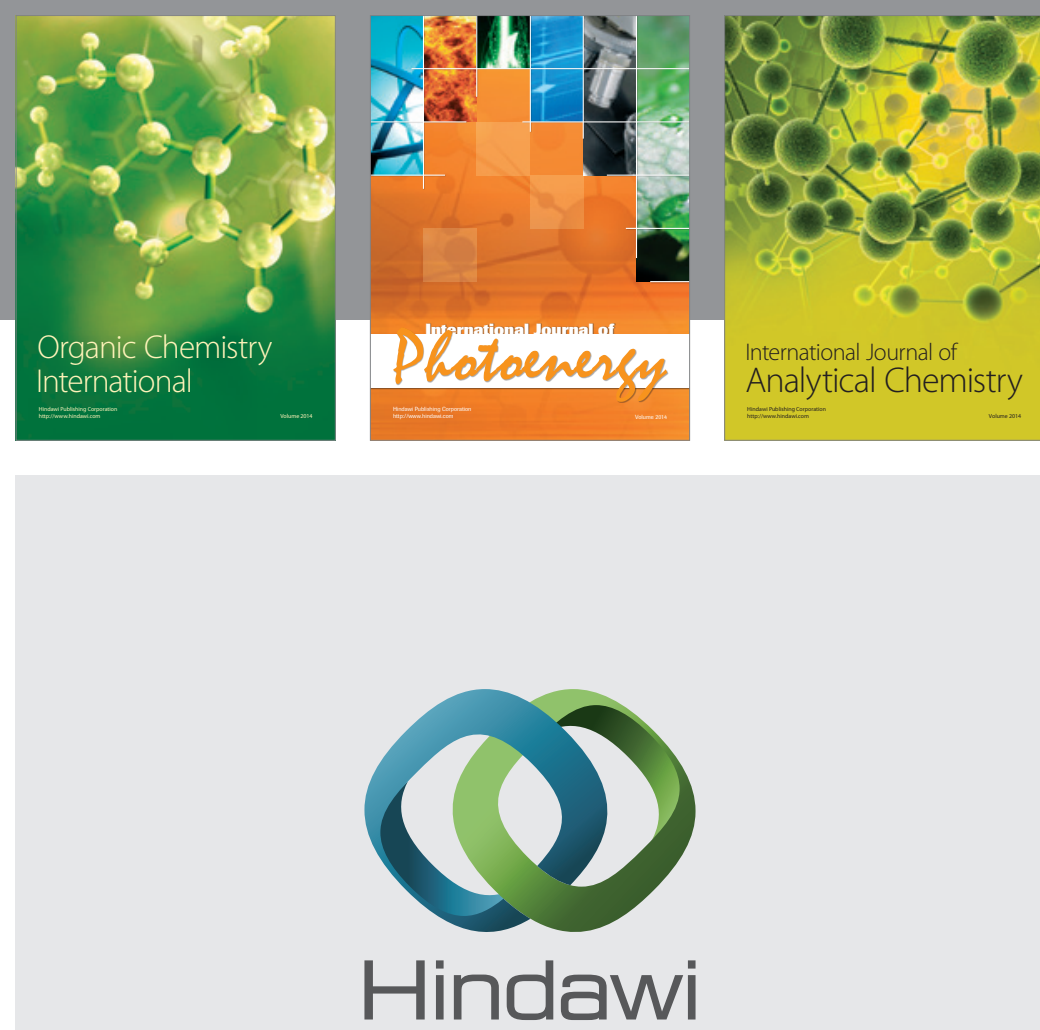

Submit your manuscripts at

http://www.hindawi.com
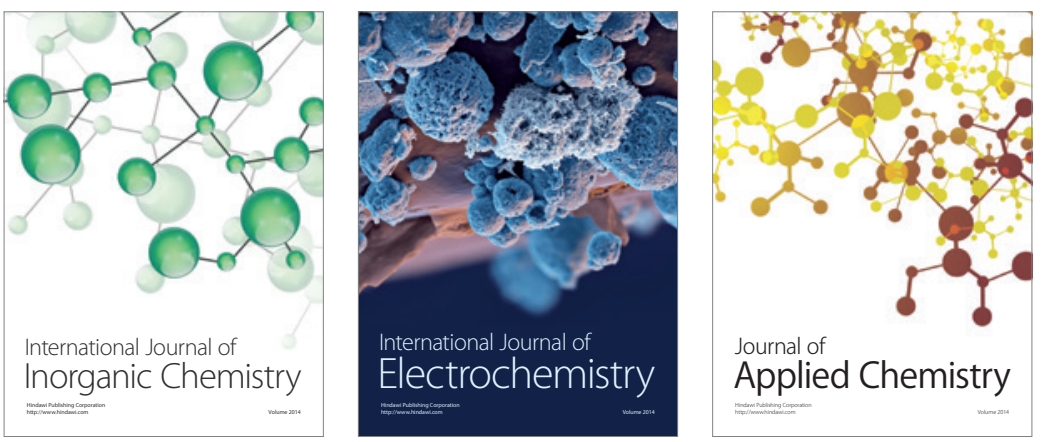

Journal of

Applied Chemistry
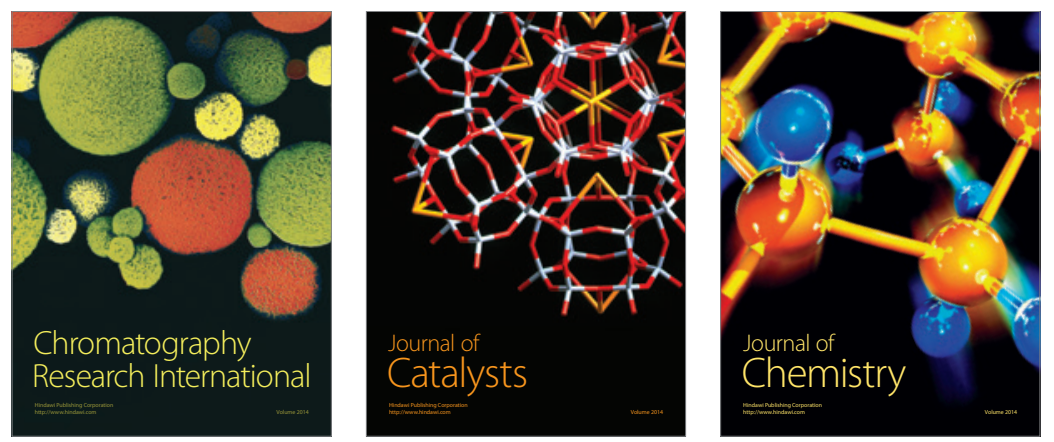
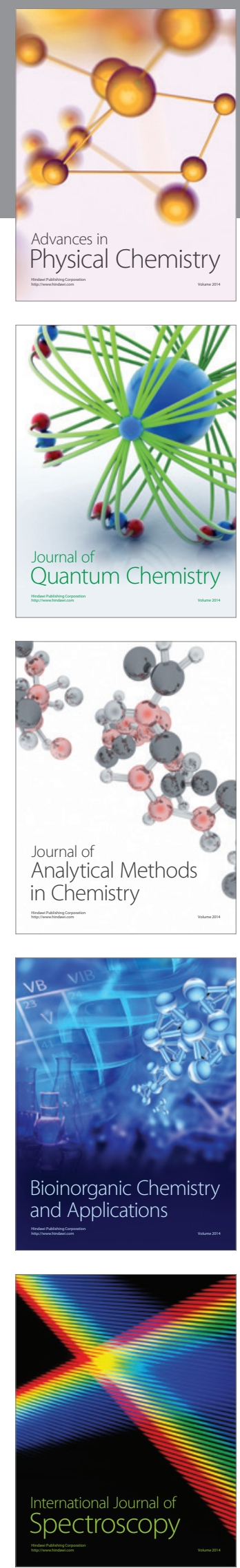